\title{
Internal Coherence
}

\begin{abstract}
This chapter offers a semiotically colored model for interrogating meaningfully interrelated events in narratives. Based on our minds' inclination to perceive gestalts (i.e., intraconnected wholes), it is suggested that meaningful temporal interrelations among represented events be understood in terms of internal coherence. It is also put forward that the internal connections in narratives can be analyzed in terms of various sorts of contiguity, forming the basis for indices that bind together the numerous parts of narratives. Several theoretical perspectives and concepts, such as narrativization, are discussed. Finally, the results are demonstrated to be valid for the phenomenon of multimodal narration, which is central for understanding transmedial narration.
\end{abstract}

Keywords Transmedial narration $\bullet$ Coherence $\bullet$ Contiguity $\bullet$ Index $\bullet$ Narrativization $\bullet$ Multimodal narration

Whereas Chap. 7 scrutinized temporally interrelated events in general and differences among temporal interrelations in particular, this chapter will move on to the last part of the definition of story as represented events that are temporally interrelated in a meaningful way. Based on the inclination of our minds to perceive gestalts, intraconnected wholes, I suggest that meaningful temporal interrelations are understood in terms of coherence. Thus, narratives are virtual spheres that, to some extent, must be 
perceived as meaningful wholes; virtual spheres that, despite possible contrasts, tensions, ambiguities, uncertainties, and even incomprehensibilities, are ultimately internally coherent enough not to fall into separate pieces.

What does it mean to say that a virtual sphere is coherent? It may be, for instance, that represented persons and actions appear to be generally interrelated; events and moods seem to somehow follow from each other rather than occur randomly; details are apprehended as parts of discernible mental or material wholes; psychological states, ideas, and concepts are developed intelligibly; physical properties are associated to material items in a consistent way; physical and psychological actions lead to reactions that are linked to the actions; emotions can be understood in the context of other emotions and activities; concepts make sense considering the setting; or that entities and developments are felt to be proportional given the overall frame.

There are no clearly discernible borders between more and less coherent virtual spheres. Coherence is partly a quality of perception involving mental parameters that cannot be measured straightforwardly. By the same token, the difference between less coherent narratives and virtual spheres representing events that are unclearly interrelated is not always clear. There may be many cracks in a virtual sphere that is still kept together, but at some point it may be felt to break.

\section{Kinds of Contiguity}

Here, I will conceptualize coherence, or more precisely internal coherence of virtual spheres, in terms of indexicality. We have already established that indices stand for objects on the ground of contiguity, to be understood as real connections, and a main function of indices in communication is to form meaningful internal interrelations. Intracommunicational indexicality is semiosis creating bonds within a virtual sphere, connecting representamens on the ground of contiguity to objects that are drawn into or formed inside the virtual sphere as it evolves.

Apart from being able to hold represented space and time, a virtual sphere is spatiotemporal in the sense that it is formed by more or less continuous perception and interpretation of physically more or less demarcated media products. Even if the perception is interrupted (as when one stops reading a book and resumes the following day), or if parts of the media products are scattered (as when communication is spread to a com- 
bination of various media types that are accessible at different points of time and places), one has the mental capacity to (re)connect the pieces so that they form a consistent virtual sphere. Hence, the sensation of the constituents of the media product, as well as what they represent, is that they are minimally co-present. As perception and interpretation evolve they are often also understood to interact.

Narrative theory often emphasizes that events in narratives are related in terms of cause-effect, which can be understood as a strong form of interaction. While it may be mechanical causality or intentional causality, the latter kind is often privileged: narration is generally restricted to the representation of actions rather than occurrences.

However, some narratological research also comprises objections to an overly strict comprehension of interaction involving one event clearly causing another, suggesting more nuanced ways of understanding cause-effect as not necessarily very direct or absolute (see for instance Branigan 1992: 26-32). It can also be noted that the notion of cause-effect is not really used in physics, where one rather reasons in terms of connected conditions: given certain circumstances, certain things will happen because of the properties of involved objects and the physical laws. If one adds a mental side to this material conceptualization, one gets a tool for investigating interrelations among narrative events that is more subtle and multifaceted than the concept of cause and effect: given certain mental and material circumstances, certain things will happen because of the properties of involved material objects and the physical laws, and because of the individual features of involved minds and the psychological commonalities. In the context of narration, naturally, all these aspects are represented and part of virtual spheres.

Co-presence and interaction are two general forms of contiguity on a scale from weaker to stronger real connections among the constituents of virtual spheres. However, one may also think in terms of more specific subclasses of contiguity, understood as particular kinds of real connections that form the ground of indices. The following list of various kinds of relational channels between entities is in no way complete and there are no clear borders between the categories; the classification is merely intended to provide a broad overview of real connections by illuminating examples ranging from material to mental contiguity.

Contiguity that does not include mental activities in the relational channel between entities includes mechanical contiguity (e.g., between finger and fingerprint, or between a bow being drawn across strings and violin sounding); electromagnetic contiguity (such as between photons 
emitted from matter and digital photographs [see Godoy 2007], or between input in a computer and what is seen on the computer screen); chemical contiguity (between photons emitted from matter and classical photographs, or between added heat and boiling water); and organic contiguity (between disease and observable symptoms, or between a dead animal and a fossil).

Contiguity that includes corporeal and conscious or unconscious mental activities in the relational channel between entities includes, for instance, the combination of mental and mechanical contiguity (e.g., between the decision to use a pencil and written text, or between sudden rage and the smashing of a window) and the combination of mental and organic contiguity (such as between emotional state and voice quality, facial expression, and body posture; between refusal to eat and sensation of hunger).

Finally, there is contiguity that merely consists of conscious or unconscious mental activities in the relational channel between entities (between sensations and assumptions; between premises and conclusions). Thus, contiguity covers everything from concrete physical connections to abstract reasoning that often ultimately derives from experiences of corporeal relations.

These differentiations of forms of contiguity offer a refined way of understanding the many possible variations of meaningfully interrelated represented objects in virtual spheres in general and more specifically meaningfully interrelated events in narratives. The general and specific subclasses of contiguity illuminate the possible grounds of both intracommunicational indexicality (internal coherence of the virtual sphere) and extracommunicational indexicality (external truthfulness; to be discussed in Chap. $9)$. In the case of internal coherence, the contiguities are virtual. In the case of external truthfulness, even indices that build on stronger real connections (i.e., interaction) and reach out to the perceived actual sphere, represent only the world as one knows it. Not only mental, but also mechanical, electromagnetic, chemical, and organic contiguity are presumed contiguities formed by collateral experience. In the end, the extracommunicational and the intracommunicational domains are both mental.

\section{NARRATIVIZATION}

The concept of perceiving virtual spheres as consisting of meaningful relations among represented events-what I subsume here under the heading of intracommunicational coherence-has already been theorized in various ways. In the discussions of gestalt psychology in Chap. 2, I noted that 
our minds crave structure and sense. This might be reformulated in terms of contiguity: we crave real connections. As contiguities can be known to us only through mental operations, they must, I repeat, be conceptualized as presumed contiguities formed by collateral experience. This comports with ideas formulated within narratological research. Seymour Chatman noticed "our powerful tendency to connect the most divergent events" (1978: 47). By acknowledging this tendency, one is not far from embracing Monika Fludernik's concept of narrativization: "making something a narrative by the sheer act of imposing narrativity on it" (Fludernik 1996: 34). A relevant factor for such acts is framing (Wolf 2014, 2017), understood as all kinds of communicative elements that set the mind of the actual communicatee toward expecting narrativity and so increase the inclination to narrativize: to construe meaningful interrelations among represented events. Thus, narrativizing is not understood here as treating series of life events as narratives but as imposing narrativity on media products.

These ideas can fruitfully be compared with a classical psychological experiment by Fritz Heider and Marianne Simmel (1944) in which a large group of people were shown a short animated movie in which two triangles and a circle move around in relation to a rectangle with an opening. The figures and their movements were not randomly chosen. Nevertheless, the moving geometrical figures were automatically seen as representations of interacting animated beings and the rectangle as a house. A majority of the participants in the experiment interpreted the interactions as a connected story. A large but far from absolute consistency in these perceived stories was also reported. The researchers emphasized "the great importance which causal interpretation plays in the organization of the events into a story" (1944: 251).

Long before the heyday of narratology, this experiment demonstrated the power of narrativization and the transmedial nature of narration. Although not discussed or even mentioned, it also demonstrated that among the cognitive schemata used by the perceivers of the animated movie, one could not only find ideas about how animated beings such as humans interact in general, by nature, but also more specific cultural conceptions regarding gender behavior; in other words, stereotypes of how men and women are and how they act. Different narrativizations used partly different schemata originating in collateral experience of both nature and culture, resulting in partly different narratives.

A follow-up experiment by Bassili (1976) established that interaction among moving geometrical figures is perceived much more strongly dur- 
ing certain spatiotemporal conditions. Therefore, it is not the case that any kinds of stimuli are capable of triggering our inclination to perceive meaningful interrelations. Narrativization requires certain properties in the media products and we do not freely narrativize just anything to the same extent. Although human minds are inventive, crave meaningful coherence, and have the power to narrativize meager material, our minds additionally have constraints - there are limits to what one perceives to be meaningfully interrelated events (Bundgaard 2007). This leads to a certain degree of intersubjectivity among actual narratees. Consequently, whereas framing may sometimes be important for construing narratives, I would argue that it is neither a necessary nor a sufficient condition for narration. On one hand, media products may have qualities that make it practically unavoidable for most perceivers to interpret in terms of narration, independently of how they are framed. On the other hand, framing cannot incline us to narrativize any random sensory configurations.

Once again, one must conclude that understanding the emergence of narratives is a question of understanding both all kinds of surrounding factors of communication and the more inherent factors of different media products.

\section{Multimodal Narration}

Presumably, the results of the experiments by Heider and Simmel (1944) and Bassili (1976) can, in principle, be applied to all kinds of media. Narrativization does not occur only in the perception of solid, twodimensional, temporal, visual, and iconic media products (cf. Neubauer 1997). It must also be expected that every change of mode of any of the media modalities may affect the way media products communicate. Another experiment, based on Heider and Simmel's animated movie, found that combining the movie with different kinds of music altered the total perceptions of narratives, to some extent (Marshall and Cohen 1988). This shows that, as most of us would probably expect, there is interaction among the various modes of media products in narration. During the last few decades, much empirical research has been dedicated to especially the interaction of sensory modes, such as vision and hearing, in communication in general.

Given that a majority of basic media types are multimodal-meaning that they have several material modes (like being partly organic, partly inorganic), several spatiotemporal modes (such as being both two- 
dimensionally spatial and temporal), several sensorial modes (being both visual and auditory, for instance), several semiotic modes (like being dominated by both iconic and symbolic signs), or several modes of more than one media modality - it is obvious that our minds have the capacity to bind these various modes together into one system of semiosis. For example, scientists know that sensory modes are connected and integrated in the brain, but know little about how this happens. Semiotic modeling, however, is more dependent on pragmatic observation than on detailed scientific evidence. Therefore, I claim that an important role of indices is to knit together the different modes of a media product so that an integrated virtual sphere, rather than a set of unrelated, mode-specific virtual spheres, can be created. Indices-representamens that call forth objects on the ground of contiguity-work effortlessly across modal borders simply because perceiving real connections among various modes is what our brains have been trained to do since the dawn of evolution. We know that solid, liquid, and gas-formed materiality interact; that our actual experience of space and time cannot be separated; and that our senses constantly cooperate to make sense of our surroundings.

On a deeper semiotic level, indices continue to connect all kinds of represented objects in a virtual sphere in order to achieve coherence. This is what I call intracommunicational indexicality: semiosis creating bonds within a virtual sphere. First, then, indices interconnect the actual perceptions of a possibly multimodal media product to prepare the formation of an integrated virtual sphere. At a later stage, indices interconnect the virtual constituents of the virtual sphere so that they can, if possible, form a coherent whole; the represented existents and events are perceived to interact. In the case of narration, indices form meaningful interrelation among virtual events in virtual time. This is the case whether the involved media products consist of a limited amount of material, spatiotemporal, sensorial, and semiotic modes (such as still images or written text) or are markedly multimodal (such as movies that are both spatial and temporal, visual and auditory, and iconic and symbolic).

\section{REFERENCES}

Bassili, John N. 1976. Temporal and spatial contingencies in the perception of social events. Journal of Personality and Social Psychology 33: 680-685.

Branigan, Edward. 1992. Narrative Comprehension and Film. London and New York: Routledge. 
Bundgaard, Peer F. 2007. The cognitive import of the narrative schema. Semiotica 165: 247-261.

Chatman, Seymour. 1978. Story and Discourse: Narrative Structure in Fiction and Film. Ithaca, NY and London: Cornell University Press.

Fludernik, Monika. 1996. Towards a 'Natural' Narratology. London and New York: Routledge.

Godoy, Hélio. 2007. Documentary realism, sampling theory and Peircean semiotics: Electronic audiovisual signs (analog or digital) as indexes of reality. Doc On-line 2: 107-117.

Heider, Fritz, and Marianne Simmel. 1944. An experimental study of apparent behavior. The American Journal of Psychology 57: 243-259.

Marshall, Sandra K., and Annabel J. Cohen. 1988. Effects of musical soundtracks on attitudes toward animated geometric figures. Music Perception 6: 95-112.

Neubauer, John. 1997. Tales of Hoffmann and others: On narrativizations of instrumental music. In Interart Poetics: Essays on the Interrelations of the Arts and Media, ed. Ulla-Britta Lagerroth, Hans Lund, and Erik Hedling, 117-136. Amsterdam and Atlanta, GA: Rodopi.

Wolf, Werner. 2014. Framings of narrative in literature and the pictorial arts. In Storyworlds across Media: Toward a Media-Conscious Narratology, ed. MarieLaure Ryan and Jan-Noël Thon, 126-147. Lincoln and London: University of Nebraska Press.

- 2017. Transmedial narratology: Theoretical foundations and some applications (fiction, single pictures, instrumental music). Narrative 25: 256-285.

Open Access This chapter is licensed under the terms of the Creative Commons Attribution 4.0 International License (http://creativecommons.org/licenses/ by $/ 4.0 /$ ), which permits use, sharing, adaptation, distribution and reproduction in any medium or format, as long as you give appropriate credit to the original author(s) and the source, provide a link to the Creative Commons licence and indicate if changes were made.

The images or other third party material in this chapter are included in the chapter's Creative Commons licence, unless indicated otherwise in a credit line to the material. If material is not included in the chapter's Creative Commons licence and your intended use is not permitted by statutory regulation or exceeds the permitted use, you will need to obtain permission directly from the copyright holder.

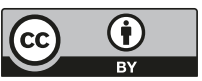

